\title{
Autoimmune polyendocrine syndrome type 1: case report and review of literature
}

\author{
Síndrome poliglandular autoimune tipo 1: \\ descrição de caso e revisão da literatura
}

Fernanda Guimarães Weiler', Magnus R. Dias-da-Silva², Marise Lazaretti-Castro'

${ }^{1}$ Bone and Mineral Unit, Division of Endocrinology, Escola Paulista de Medicina, Universidade Federal de São Paulo (Unifesp/ EPM), São Paulo, SP, Brazil ${ }^{2}$ Biochemistry Department Unifesp/EPM, São Paulo, SP, Brazil

The authors invite colleagues to enroll patients with APS-1 in the Brazilian clinical and molecular study carried out in our institution

Correspondence to: Fernanda Guimarães Weiler Unidade de Doenças Osteometabólicas,

Universidade Federal de São Paulo Rua Borges Lagoa, 800 04038-001 - São Paulo, SP, Brazil fgweiler@unifesp.br

Received on 12/July/2011 Accepted on 3/Dec/2011

\begin{abstract}
SUMMARY
Autoimmune polyendocrine syndrome type 1 (APECED) is a rare autosomal recessive disorder characterized by autoimmune multiorgan attack. The disease is caused by mutations in the autoimmune regulator gene $(A I R E)$, resulting in defective AIRE protein, which is essential for selftolerance. Clinical manifestations are widely variable. Although the classic triad is composed by mucocutaneous candidiasis, hypoparathyroidism and adrenal failure, many other components may develop. Treatment is based on supplementation of the various deficiencies, and patients require regular follow-up throughout their lifespan. This article describes the case of a patient with the disease, and reviews literature data on the epidemiology, clinical course, immunogenetic aspects, diagnosis and treatment of the syndrome. Arq Bras Endocrinol Metab. 2012;56(1):54-66
\end{abstract}

\section{SUMÁRIO}

Síndrome poliglandular autoimune tipo 1 é uma rara desordem autossômica recessiva caracterizada por ataque autoimune a diversos órgãos. A doença é causada por mutações no gene AIRE (autoimmune regulator), resultando em uma proteína AIRE defeituosa, proteína esta essencial para a manutenção da autotolerância. As manifestações clínicas são extremamente variáveis. A tríade clássica é composta por candidíase mucocutânea crônica, hipoparatiroidismo e insuficiência adrenal, porém diversos outros componentes podem estar presentes. A base do tratamento é a reposição das diversas deficiências, e os pacientes devem ser acompanhados por toda a vida. Este artigo descreve o caso de uma paciente com a síndrome e apresenta uma revisão sobre a epidemiologia, quadro clínico, aspectos imunogenéticos, diagnóstico e tratamento da desordem, de acordo com a literatura publicada. Arq Bras Endocrinol Metab. 2012;56(1):54-66

\section{INTRODUCTION}

A utoimmune polyendocrine syndromes (APS) are rare disorders characterized by the coexistence of at least two endocrine gland insufficiencies mediated by autoimmune mechanisms (1). Additionally, nonendocrine immune diseases may be present. According to the Neufeld and Blizzard Classification of 1980 (2), there are four main types of APS. Their most relevant features are described in table 1.

APS type 1, also known as APECED (autoimmune polyendocrinopathy-candidiasis-ectodermal dystrophy), MEDAC (multiple endocrine deficiency autoimmune candidiasis syndrome), juvenile autoimmune polyendo- crinopathy, or Whitaker's syndrome (OMIM 240300), is classically defined by the association of at least two of three major component diseases: chronic mucocutaneous candidiasis, primary hypoparathyroidism, and autoimmune adrenal insufficiency (2-4). When relatives of a patient are analyzed, only one condition is required for APS-1 diagnosis (5). Recently, some authors have proposed that genetic studies and anti-interferon (IFN) antibodies (Abs) should be included as diagnostic criteria $(6)$.

The first description of the association between hypoparathyroidism and candidiasis was reported by Thorpe and Handley in 1929 (7). However, adrenal insufficiency was not related to the syndrome until 1946 (8). 
Table 1. Characteristics of the autoimmune polyendocrine syndromes (APS)

\begin{tabular}{ll}
\hline APS type 1 & $\begin{array}{l}\text { Chronic candidiasis, hypoparathyroidism, } \\
\text { autoimmune adrenal insufficiency (at least two of them should } \\
\text { be present) }\end{array}$ \\
APS type 2 & $\begin{array}{l}\text { Autoimmune adrenal insufficiency (must always be present) + } \\
\text { autoimmune thyroid disease and/or type } 1 \text { diabetes mellitus }\end{array}$ \\
APS type 3 & $\begin{array}{l}\text { Autoimmune thyroid disease + other } \\
\text { autoimmune diseases (excluding autoimmune adrenal } \\
\text { insufficiency, hypoparathyroidism, chronic candidiasis) }\end{array}$ \\
APS type 4 & $\begin{array}{l}\text { Two or more organ-specific autoimmune diseases (which do not } \\
\text { fall into type 1,2, or 3) }\end{array}$ \\
\hline
\end{tabular}

\section{EPIDEMIOLOGY}

The syndrome is rare, and approximately 500 patients have been reported worldwide. The highest prevalence was found among the Iranian Jewish community $(1: 9,000)(9)$, in Sardinia $(1: 14,400)(10)$ and in Finland $(1: 25,000)(4)$. There is no published data about the prevalence of APS- 1 in Brazil.

In most cases, APS-1 appears in infancy or childhood $(3,4,11,12)$, and the three major component diseases develop in chronological order: candidiasis first, commonly appearing before the age of 5 ; then hypoparathyroidism before the age of 10; and finally, adrenal insufficiency, before the age of 15 (4,11-13). One report showed that the complete triad was present only in $50 \%$ of patients at the age of $20,55 \%$ at age 30 , and $40 \%$ at age 40 (14), and numbers were similar in other reports $(4,11)$. Studies revealed that the earlier the first manifestation appears, the greater the number of disease components $(3-5,11)$. The female-to-male ratio varies in different reports, from 0.8:1 to 2.4:1 (2-4,11).

\section{CASE REPORT}

A 28-year-old woman, born to consanguineous parents, first presented nail candidiasis and blurred vision at age 5 . At the age of 17, she developed paresthesia and muscle cramps and, by that time, candidiasis had already spread to the skin along her legs and trunk. Three years later, diarrhea developed in association with severe weakness and worsening tetany. Serum levels of calcium were extremely low and PTH was undetectable. The patient was then admitted in the medicine service at the Universidade Federal de São Paulo, where it was noted that, besides hypoparathyroidism and mucocutaneous candidiasis, she presented primary amenorrhea with incomplete pubertal development, nail dystrophy, dental enamel hypoplasia, and corneal keratopathy (Figure 1). Laboratory tests excluded celiac disease, cystic fibrosis, failure of exocrine pancreas, or intestinal infections as causes of the chronic diarrhea. Further investigations revealed subclinical adrenal insufficiency, pernicious anemia, renal tubulopathy, and impaired fasting glucose. Antibodies against pancreatic glutamic acid decarboxylase 65 (GAD65), but not against IA-2 and insulin, were positive. Although respiratory symptoms were not present, chest computed tomography scan revealed bilateral bronchiectasis. The patient is currently under treatment with anti-fungal medication and glucocorticoid, estrogen, and vitamin B12 replacement. Calcium serum levels remain low, even with the administration of high doses of calcium supplement and vitamin D derivatives, probably due to malabsorption. The patient was submitted to resection of the right cornea and transplantation of her own conjuntival cells cultured on amniotic membrane, with good results. Now, the procedure is scheduled to be performed in her left eye.

\section{CLINICAL MANIFESTATIONS OF APS-1}

Cbronic mucocutaneous candidiasis (CMC) - Chronic superficial infection with Candida albicans is the most common manifestation in APS-1. It is present in almost $100 \%$ of the patients, with the exception of Iranian Jews, in whom the infection is seldom reported $(4,9,12,15)$. In most cases, CMC is the first of the major components of APS- 1 to appear, often occurring before age 5 $(4,11,12)$, and its severity is variable. It preferably affects the oral mucosa causing a mild form of intermittent angular cheilitis. More severe cases include inflammation of most of the oral mucosa, hyperplastic CMC with thick white or grey plaques of yeast and hyperkeratosis, and atrophic form with thin mucosa and leukoplakic areas. The latter are especially troublesome because of their carcinogenic potential. Esophageal mucositis with substernal pain and dysphagia may occur. Squamous cell carcinoma of the mouth or esophagus shows an obscure prognosis. Symptomatic intestinal candidiasis includes abdominal pain, flatulence and diarrhea. Female patients may suffer from vulvovaginitis. Skin and nails may be affected but, in the majority of cases, the infection is limited to no more than $5 \%$ of the skin surface. Generalized candidiasis has only been reported in patients on immunosuppressive medication $(4,11,12)$.

Hypoparathyroidism (HP) - HP is usually the first endocrine disease to develop during the course of APS- 
$\mathrm{l}$ and it has been reported in 70\%-93\% of the cases (2$4,11,12,16)$. It varies according to gender, affecting $98 \%$ of female patients, but only $71 \%$ of male patients (17). Interestingly, when adrenal insufficiency is the first endocrinopathy, susceptibility to HP appears to be reduced $(12,17)$. Many symptoms of hypocalcemia are nonspecific, such as paresthesias and muscle cramps. Seizures may occur in more severe cases. Hypomagnesemia is common and may complicate the condition (12).

Primary adrenal insufficiency (AI) or Addison's disease - The prevalence of AI is $60 \%-100 \%$, with peak incidence around 12 years of age $(2-4,11,16)$. It is a lifethreatening condition that should be rapidly recognized and treated. Symptoms are fatigue, weight loss, salt craving, hypotension, abdominal pain and increased pigmentation of the skin. It is important to be aware that hypocortisolism and hypoaldosteronism may appear years apart.

Hypergonadotropic hypogonadism - Hypergonadotropic hypogonadism appears in $12 \%-60 \%$ of the APS1 patients $(2-4,11,16)$, with a prevalence three times higher among females $(2,4)$, possibly due to the bloodtestis barrier that protects Leydig cells from an autoimmune attack. Half of the affected female patients have primary amenorrhea with absence or early interruption of spontaneous pubertal development, and others develop premature menopause (12).

Autoimmune thyroid diseases - Hypothyroidism is relatively uncommon, affecting no more than $30 \%$ of the APS-1 patients. It develops more often following puberty and by middle age, usually before the age of 30 $(4,9,11,12)$. Hyperthyroidism is extremely rare (12). A higher frequency of autoimmune thyroiditis is observed in patients with APS- 1 caused by a specific mutation, the G228W missense mutation (18).

Type 1 diabetes mellitus (TID) - TID has been described in up to $18 \%$ of the APS-1 subjects. The highest prevalence was seen in Finnish patients $(2-4,11,19)$. This syndrome is part of the heterogeneous sprectrum of TID $(20,21)$.

Pituitary failure and lymphocytic bypophysitis Single or multiple pituitary defects have occasionally been described in APS-1. Growth hormone deficiency is the most commonly reported defect, but central diabetes insipidus and gonadotropin or ACTH deficiencies have also been seen. Autoimmune hypophysitis rarely affects patients $(4,9,11,12,22)$.

Chronic atrophic gastritis and pernicious anemia - Autoimmune gastritis is characterized by gastric mucosa atrophy, selective loss of parietal cells from the gas- tric mucosa, and submucosal lymphocyte infiltration. Vitamin B12 absorption may be impaired, leading to pernicious anemia $(2,4,9,11,12)$.

Intestinal dysfunction and malabsorption - The prevalence of intestinal dysfunction varies from 15 up to $22 \%(3,4,11)$. The symptoms consist of chronic or periodic constipation, chronic or recurrent diarrhea and malabsorption, which cause weight loss, growth retardation and erratic absorption of medications. Although it has been considered a nonendocrine manifestation, new evidence indicate that an autoimmune attack against cells of the gastrointestinal endocrine system actually occurs in APS-1 (see the SELF-ANTIBODIES section, below). Besides, malabsorption may be attributed to a variety of causes, such as celiac disease, cystic fibrosis, failure of exocrine pancreas, intestinal infections (Candida, Giardia lamblia and Clostridium difficile), and intestinal lymphangectasia $(11,12,22)$. In some cases, diarrhea is caused or worsened by the presence of $\mathrm{HP}$, since hypocalcemia may cause a functional deficit in cholecystokinin (23). Then, a vicious cycle sets in: hypocalcemia causes malabsorption, which impairs the absorption of calcium and vitamin $\mathrm{D}$, worsening the hypocalcemic state.

Autoimmune hepatitis - Hepatitis has been described in $8 \%-26 \%$ of the cases $(3,4,11)$. Severity is variable, ranging from an asymptomatic course with spontaneous regression, to fulminant necrotizing disease with extremely high mortality $(4,11,12)$.

Spleen aplasia or bypoplasia - This disorder is present in about $10 \%$ of children and $20 \%$ of adults (12). It appears to be due to a progressive autoimmune-mediated destruction or vascular insult. Asplenia compromises the immune response to encapsulated bacteria, increasing the susceptibility to Pneumococcus infections. Suspicion of asplenia can be raised by the presence of Howell-Jolly bodies, thrombocytosis, anysocites, poikylocytes, target cells, and burr cells on peripheral blood smears (24).

Renal disease - Tubulointerstitial nephritis has been described, sometimes with progression to terminal renal failure and need for kidney transplantation $(12,25)$.

Pulmonary disease - Pulmonary disease has been sporadically observed in APS-1 patients as primary pulmonary hypertension (26), or autoimmune bronchiolitis and bronchiectasis (27).

Ectodermal dystrophy - The components of ectodermal dystrophy are keratoconjunctivitis, dental enamel 
hypoplasia and punctate nail defects. The pathomechanism is unknown.

- Keratoconjunctivitis appears in $10 \%-35 \%$ of patients $(3,4,11,12)$, usually before the age of $5(4,11,12)$. It is extremely rare among Iranian Jewish patients (9). Affected individuals suffer from intense photophobia, blepharospasm, lacrimation, and blurred vision. Progression to blindness may occur. Corneas show irregular confluent opacities, with bulbar injection of the conjunctiva, and subsequent superficial corneal neovascularization (28).

- Enamel hypoplasia of permanent teeth is observed in approximately $80 \%$ of the patients (4). This defect predisposes them to further damage and cavities.

- Punctate nail dystrophy was also described in APS-1 patients $(4,11)$.

Vitiligo -Vitiligo may develop since the first month after birth and is present in $8 \%-25 \%$ of the patients. Extent is highly variable, from spots to almost all corporal surfaces $(2-4,11,12)$.

Alopecia - Another component of the syndrome is alopecia, which may appear since the first years, with increasing prevalence to $40 \%$ by middle age. It involves the scalp, eyelashes, eyebrows, axilla, and pubis. Alopecia may be present as transient hairless scalp patches, but it may become extensive $(11,12)$.

Vasculitis - Recurrent maculopapular, morbilliform or urticarial erythema with fever has also been seen in APS-1. Skin biopsies performed in some cases revealed lymphoplasmacytic vasculitis $(4,11)$.

Other clinical manifestations -Cholelithiasis (24), iridocyclitis, optic nerve atrophy, retinal degeneration (29), calcified plaques of the tympanic membranes $(4,14)$, selective IgA deficiency, hypergammaglobulinemia (4), hemolytic anemia $(11,12)$, hypoplastic anemia (12), autoimmune thrombocytopenia (25), scleroderma $(11,14)$, Sjögren syndrome $(11,12,29)$, lichen planus $(11,14)$, hypermineralocorticoidism-like state $(12,14)$, reversible metaphyseal dysplasia (30), progressive myopathy (31).

\section{THE AIRE GENE AND ITS ENCODED PROTEIN}

APS-1 results from defects in the autoimmune regulator (AIRE) gene (Gen Bank accession Z97990), which was identified and cloned simultaneously by two groups in 1997 (32,33). The gene, located on chromosome $2 \mathrm{lq} 22.3$, is approximately $13 \mathrm{~kb}$ in length, and is composed of 14 exons (NCBI RefSeqGene NG_009556.1) (Figure 2).
AIRE encodes a 545 -amino acid protein with molecular weight of approximately $55-58 \mathrm{kDa}$, which acts as a transcriptional regulator binding to DNA as dimer and tetramer, but not as a monomer. It strongly binds to G-doublets in an A/T-rich environment where the preferred motif is a tandem repeat such as ATTGGTTA, and/or combined with a TTATTA-box motif $(34,35)$. This protein contains several structural motifs that are typical of nuclear proteins and transcription factors (Figure 3).

The homogeneously staining region (HSR) at the amino-terminal end of the molecule mediates homodimerization (35). A caspase recruitment domain (CARD), which is needed for the transactivation activity of AIRE, overlaps with the homogeneously staining region (36). Adjacent to the HSR is a nuclear localization signal (NLS) (37) followed by a SAND domain ( Spl00, AIRE-1, NucP4l/75, DEAF-1), with DNAbinding properties (34). There are four interspersed LXXLL motifs, typical of nuclear receptor binding proteins, where protein-protein interactions are thought to occur (38). However, in AIRE, the significance of these domains is not totally determined. AIRE also contains two zinc fingers of the plant homeodomain type (PHD) located in the $\mathrm{C}$ terminus $(32,33)$. PHD domains are restricted to nuclear transcriptional regulators, including coactivators and chromatin modulating proteins (39). It has been shown that the first PHD (PHDl) of AIRE acts as an E3 ubiquitin ligase, mediating the transfer of ubiquitin to specific proteins (40), a process involved in innate and adaptive immunity and a variety of other cell processes (Figure 4). However, other functional assessments did not support those findings (41). A proline-rich region (PRR) that may be involved in protein-protein interactions, is located between the two PHD domains.

\section{THE ROLE OF AIRE IN AUTOIMMUNITY}

The AIRE gene is largely expressed in the thymus, but its protein is also found in lymph nodes, spleen, fetal liver, and peripheral blood cells $(33,37,42,43)$. Within the thymus, AIRE plays an important role in induction of T-cell tolerance. The gene is expressed primarily in medullary thymic epithelial cells (mTEC), where it promotes ectopic expression of several peripheral tissue-restricted self-proteins, resulting in the presentation of the self-antigens (Ags), and clonal deletion of the T-cells with high affinity for such Ags (42). In addi- 
tion, AIRE is a proapoptotic factor, expressed at the final maturation stage of mTECs. Evidence suggests that T-cells can also recognize self-Ags indirectly on dendritic cells that have enfolded AIRE-induced apoptotic mTECs or mTEC fragments (44). The absence of the AIRE protein results in a defective negative selection of self-reactive T-cells, which escape to the periphery, causing a multiorgan attack (Figure 5).

Beyond the control of negative selection of self-reactive $\mathrm{T}$ cells, additional mechanisms of AIRE-dependent tolerance have been investigated. One of these mechanisms is the positive selection of regulatory T-cells (Treg), specialized cells that control self-reactive T-cells and maintain immune system homeostasis. A study showed a lower level of circulating $\mathrm{CD} 4^{+}{ }^{+}$Foxp $3^{+}$Treg in some APS-1 patients (45). Opposed to this finding, this last study cited, and some other studies with Aire-deficient mice did not report differences in absolute numbers, relative frequency, and suppressive function of Treg $(45,46)$. Thus, the effect of AIRE deficiency on Treg production and function still remains to be determined.

AIRE may also control peripheral dendritic cells (DC) maturation and function. Indeed, researchers have noted abnormalities in peripheral DC function in APECED patients (47), although defects were generally modest, and sometimes contradictory (48).

Protection against Candida is thought to be T-cell mediated, and Thl7-associated cytokines, such as IL17A, IL-17F, and IL-22, may be implicated in the defense against this fungal infection (49). Recent studies in APS- 1 patients published that CMC was associated with self-antibodies (Abs) against those Thl7-associated cytokines $(50,51)$, and reported defective IL-17F and IL-22 responses to Candida albicans Ags (51). It seems that there is a normal $\mathrm{B}$-cell response to Candida, which prevents the development of systemic candidiasis.

\section{GENETIC ASPECTS}

Most frequently, the syndrome displays an autosomal recessive inheritance, but a unique autosomal dominant mutation of AIRE has been described in an Italian family (18). Study in mice and functional analysis of such mutation confirmed that the encoded protein has a dominant-negative effect (52).

To date, more than 70 different mutations of the AIRE gene have been identified (Human Gene Mutation Database - http://www.hgmd.cf.ac.uk), ranging from a single nucleotide change to large deletions, distributed throughout the gene. Another helpful site for updating the mutations is the AIREbase: mutation registry for autoimmune polyendocrinopathy candidiasis-ectodermal dystrophy (APECED) (http://bioinf.uta.fi/AIREbase/). Table 2 shows coding variations in AIRE gene.

The most common mutations are the nonsense mutation R257X (present in more than $80 \%$ of the Finish disease alleles) $(32,33)$; the $\mathrm{Y} 85 \mathrm{C}$ missense mutation (frequently found among the Iranian Jewish community) (53); the nonsense mutation Rl39X (the most common in Sardinia) (10); and the deletion 967-979dell3 (present in Norwegians, British and North-Americans) (54-56). In addition, an unpublished personal communication of Moraes-Vasconcelos pointed out that the latter is also the most common mutation among South-Americans.

Although APS- 1 is considered an autosomal recessive disease, few cases show only one defective allele, whereas in other reports not a single mutant allele was identified in typical APS-1 patients $(15,57)$. As already stated above, only one autosomal dominant mutation of AIRE has been described up today, the missense mutation G228W (18). Some mutations affecting splicing sites have also been reported (56).

Notably, different mutations have not been consistently correlated with specific phenotypes. Individuals with exactly the same mutations (even siblings) present wide variations in clinical manifestations and course of the disease $(4,12)$. Some authors observed exceptions and reported few phenotype-genotype associations: the R257X mutation is linked to a higher frequency of mucocutaneous candidiasis; lower prevalence of candidiasis and Addison's disease are found in the Iranian Jewish patients with the missense mutation $\mathrm{Y} 85 \mathrm{C}$; and alopecia is more common in individuals with deletion 967-979dell3 $(9,50)$. Autoimmune thyroiditis is usually seen in individuals carrying the G228W missense mutation (18). It seems evident that the allelic heterogeneity of the AIRE gene provides very little explanation on the variation of the phenotype. Therefore, other modifying factors may be involved. Environmental factors clearly contribute to the variation in the manifestations, but there is evidence that the phenotype is modified by other genetic elements.

Since certain alleles in the genes of human leukocyte antigen (HLA) class I (A, B, Cw) and class II (DR, DQ, $\mathrm{DP})$ are strongly associated with autoimmune diseases, such as type 1 diabetes and autoimmune thyroiditis, several studies have been performed to define whether they have an impact on APS-1. In a study with APS-1 patients 


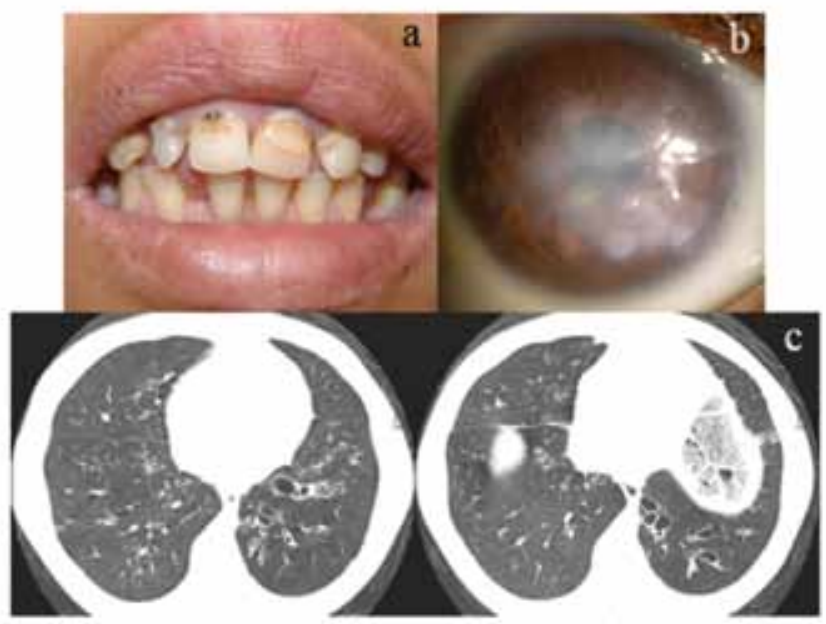

a) Dental enamel hypoplasia; b) Corneal opacity; c) Bronchiectasis.

Figure 1. Manifestations of APS1.
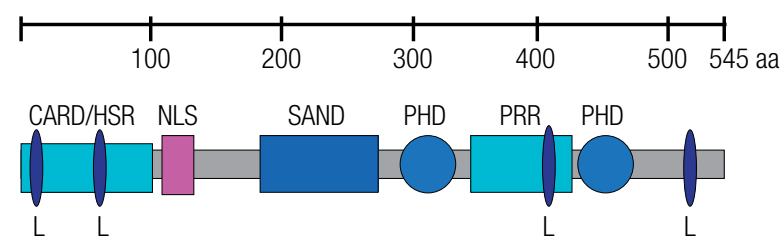

CARD: caspase recruitment domain; HSR: homogeneously staining region domain; NLS: nuclear localization signal; SAND: Sp100, AIRE-1, NucP41/75, DEAF-1; L (LXXLL): nuclear receptor-binding motifs; PHD: plant homeodomain type zinc fingers; PRR: Proline-rich region.

Figure 3. Schematic representation of the AIRE protein and its functional domains.

A

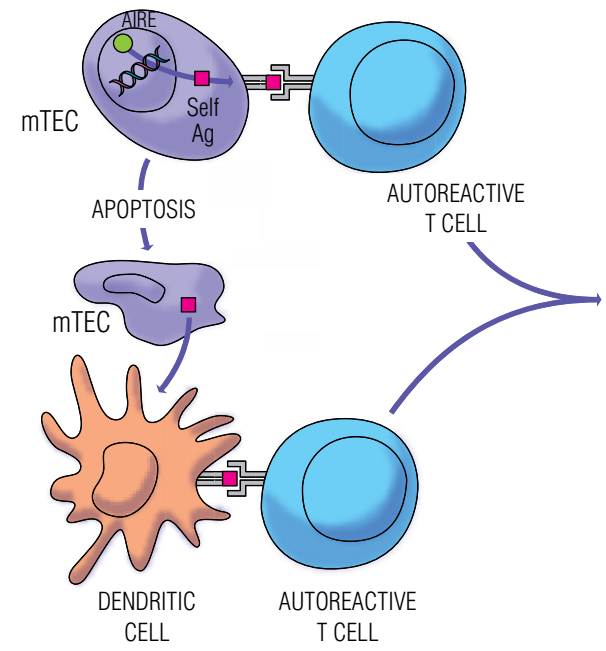

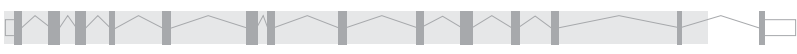

Figure 2. AIRE gene depicted in 14 exons where most frequent mutations are placed. (http://www.ensembl.org/Homo_sapiens/Transcript/Summary? $\mathrm{db}=$ core; $g=$ ENSG00000160224).

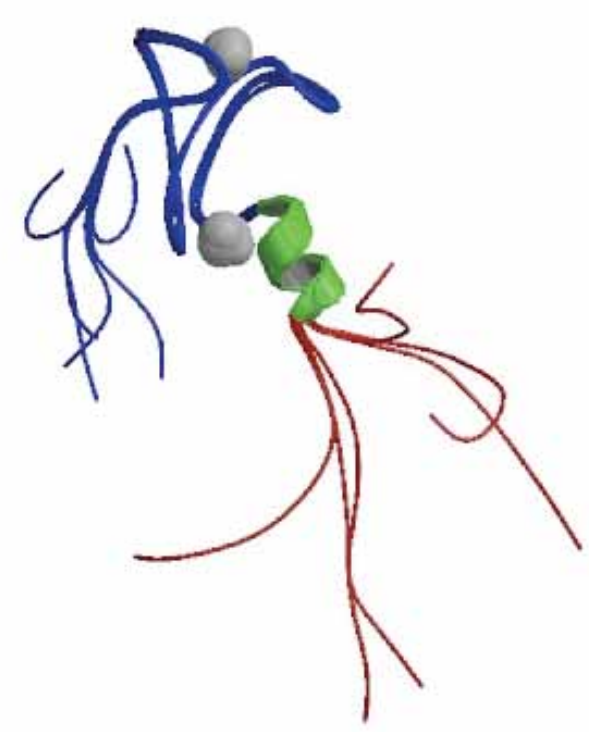

Figure 4. 3D NMR-structure of the first PHD finger of AIRE1 protein, resolved by Bottomley, et al. 2005 (41).
B

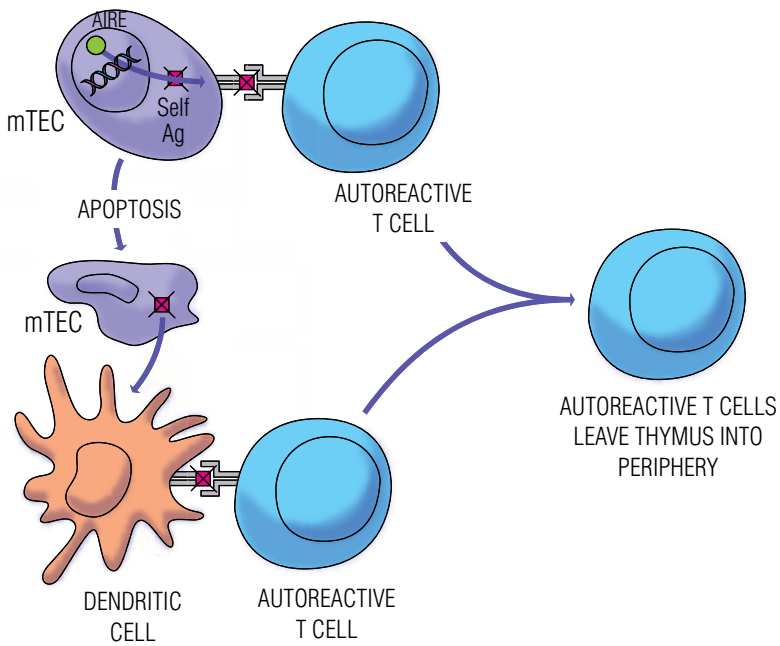

Figure 5. The role of AIRE in negative selection of autoreactive T cells. A) Normal function; B) APS-1. 
Table 2. Synonymous and non-synonymous coding variations in AIRE. Registered SNP (rs) identification and its functional impact on transcriptional factor function

\begin{tabular}{|c|c|c|c|c|c|}
\hline Residue & Variation ID & Type of mutation & Changed residues & Mutated codon & Functional study \\
\hline 1 & rs 121434258 & Non-synonymous coding & $M, L$ & ATG, TTG & Benign \\
\hline 33 & rs3746964 & Synonymous coding & A & GCT, GCC & - \\
\hline 83 & rs121434255 & Non-synonymous coding & $K, E$ & AAG, GAG & Probably damaging \\
\hline 101 & rs117659629 & Non-synonymous coding & P, S & CCC, TCC & Probably damaging \\
\hline 116 & rs74162060 & Synonymous coding & $P$ & CCG, CCA & - \\
\hline 139 & rs121434256 & Stop gained & $\mathrm{R}$, * & CGA, TGA & - \\
\hline 143 & rs41276642 & Non-synonymous coding & $P, L$ & CCA, CTA & Benign \\
\hline 166 & rs11910214 & Non-synonymous coding & $P, L$ & CCG, CTG & Possibly damaging \\
\hline 179 & rs72650674 & Synonymous coding, Splice site & $\mathrm{N}$ & AAC, AAT & - \\
\hline 183 & rs34219046 & Non-synonymous coding & T, N & ACC, AAC & Probably damaging \\
\hline 196 & rs878081 & Synonymous coding & $S$ & TCC, TCT & - \\
\hline 199 & rs74162061 & Non-synonymous coding & V,I & GTC, ATC & Possibly damaging \\
\hline 227 & rs1055311 & Synonymous coding & G & GGC, GGT & - \\
\hline 228 & rs121434257 & Non-synonymous coding & $G, W$ & GGG, TGG & Probably damaging \\
\hline 252 & rs34397615 & Non-synonymous coding & $P, L$ & CCG, CTG & Benign \\
\hline 257 & rs121434254 & Stop gained & $\mathrm{R}$, * & CGA, TGA & - \\
\hline 266 & rs41277550 & Synonymous coding, Splice site & $P$ & CCC, ССТ & - \\
\hline 278 & rs1800520 & Non-synonymous coding & $S, R$ & $A G C, A G G$ & Benign \\
\hline 291 & rs56179981 & Synonymous coding & L & CTC, CTT & - \\
\hline 301 & rs56393821 & Non-synonymous coding & $\mathrm{V}, \mathrm{A}$ & GTG, GCG & Benign \\
\hline 309 & rs74162062 & Non-synonymous coding & $\mathrm{I}, \mathrm{M}$ & ATC, ATG & Possibly damaging \\
\hline 328 & rs74162063 & Non-synonymous coding & $\mathrm{R}, \mathrm{W}$ & CGG, TGG & Probably damaging \\
\hline 332 & rs59281354 & Non-synonymous coding, Splice site & $S, T$ & AGT, ACT & Probably damaging \\
\hline 372 & rs61737006 & Synonymous coding & S & TCG, TCA & - \\
\hline 373 & rs79212994 & Non-synonymous coding & $A, V$ & GCG, GTG & Benign \\
\hline 399 & rs1800521 & Synonymous coding & A & GCT, GCC & - \\
\hline 401 & rs61737072 & Synonymous coding & $P$ & ССТ, ССС & - \\
\hline 405 & rs72650676 & Synonymous coding & $\mathrm{P}$ & CCG, CCA & - \\
\hline 416 & rs74162064 & Synonymous coding & $P$ & CCC, CCG & - \\
\hline 441 & rs72650677 & Non-synonymous coding & $\mathrm{T}, \mathrm{M}$ & ACG, ATG & Probably damaging \\
\hline 460 & rs55840791 & Non-synonymous coding & $P, S$ & CCA, TCA & Benign \\
\hline 468 & rs7281600 & Synonymous coding & $\mathrm{T}$ & ACG, ACA & - \\
\hline 471 & rs74203920 & Non-synonymous coding & $\mathrm{R}, \mathrm{C}$ & CGC, TGC & Probably damaging \\
\hline 492 & rs72650679 & Synonymous coding & $P$ & CСС, ССТ & - \\
\hline 526 & rs1133779 & Synonymous coding & D & GAT, GAC & - \\
\hline
\end{tabular}

* Stop codon.

from 12 different countries, Addison's disease was positively associated with the HLA-DRBI * 03 allele (relative risk RR 8.8). Alopecia was also found to be significantly and positively associated with the HLA-DQBI *0302 (RR 6.6) and $\mathrm{DRBl}^{*} 04$ (RR 4.8). The most common protective alleles for $\mathrm{TlD}, \mathrm{DRBl}$ * 15 and $\mathrm{DQB1}$ *0602, appeared similarly protective in APS-1 patients. Several other weaker associations were found, but their true significance is not known (19). In fact, some HLA polymorphisms seem to influence the APECED phenotype, although those associations appear to be weaker than in common autoimmune diseases. 


\section{SELF-ANTIBODIES}

The autoimmune destructive process is presumed to be T-cell mediated, but typically patients display a variety of self-Abs against proteins present in the affected organs, frequently intracellular enzymes (Table 3 ). The role of such self-Abs in the destruction remains unclear, but they are useful diagnostic markers, and their appearance usually precedes the development of the clinical manifestation.

Table 3. Relevant self-antigens in APS-1, and corresponding diseases

\begin{tabular}{|c|c|}
\hline APS-1 components & Antigens \\
\hline Adrenal insufficiency & $21-\mathrm{OH}, 17 \alpha-\mathrm{OH}, \mathrm{SCC}$ \\
\hline Hypogonadism & scc, $17 \alpha-0 H$, TSGA10 \\
\hline Hypoparathyroidism & NALP5, CaSR \\
\hline Hypothyroidism & $\mathrm{TPO}, \mathrm{Tg}$ \\
\hline Type 1 diabetes & GAD65, IA-2, insulin \\
\hline Gastrointestinal dysfunction & TPH, HDC, GAD65 \\
\hline Immune hepatitis & CYP1A2, CYP2AC, AADC, TPH, HDC \\
\hline Immune gastritis & $\mathrm{H}^{+} / \mathrm{K}^{+}$ATPase intrinsic factor \\
\hline Lung disease & KCNRG \\
\hline Vitiligo & Melanocyte, SOX9, SOX10, AADC \\
\hline Alopecia & $\mathrm{TH}$, hair follicles \\
\hline Candidiasis & IL -17A, IL-17F, IL-22 \\
\hline
\end{tabular}

21-OH: 21-hydroxylase, $17 \alpha-0 H$ : 17 $\alpha$-hydroxylase, scc: side-chain cleavage enzyme, NALP5: NACHT leucine-rich-repeat protein 5, CaSR: calcium-sensing receptor, TPO: thyroid peroxidase, Tg: thyroglobulin, GAD65: glutamic acid decarboxylase 65, IA-2: protein tyrosine phosphatase, TPH: tryptophan hydroxylase, HDC: histidine decarboxylase, CYP1A2 and CYP2AC: cytochromes P450 1A2 and 2A6, AADC: aromatic l-amino acid decarboxylase, TH: tyrosine hydroxylase.

Self-Abs against the steroidogenic enzymes 21-hydroxylase $(21-\mathrm{OH})$, side-chain cleavage enzyme (scc) and $17 \alpha$-hydroxylase $(17 \alpha-\mathrm{OH})$ may be found in APS1 patients with Addison's disease, and at least one of these Abs is present in more than $80 \%$ of the patients $(16,58)$. A study with 90 patients revealed a strong association between self-Abs against $21-\mathrm{OH}$, scc, and adrenal insufficiency, with odds ratio of 7.8 and 6.8 , respectively. The $17 \alpha-\mathrm{OH} \mathrm{Ab}$ was not shown to be an independent marker in this case (16).

Self-Abs against scc and $17 \alpha-\mathrm{OH}$ have been implicated in APS-1, as well as gonadal self-Ags, especially among female patients (58). Self-Abs against scc, but not $17 \alpha-\mathrm{OH}$, were associated with hypogonadism (16). Researches revealed that the testis-expressed protein TSGAl0 is also a target of immune reactions in male hypogonadal patients (59).

Parathyroid self-Ags targeted by self-Abs in APS-1 patients have been identified as the NACHT leucine- rich-repeat protein 5 (NALP5) (60) and the extracellular domain of the calcium-sensing receptor (CaSR) (61). However, other studies have not confirmed CaSR as relevant self-Ag $(16,60)$. It seems that the detection of CaSR Abs is critically dependent on the type of assay, as revealed by a study that analyzed APS-l patient sera for the presence of CaSR Abs using different assays (61). Immunoprecipitation detected CaSR Abs in $85.7 \%$ of the APS-1 patients, while flow cytometry detected CaSR $\mathrm{Ab}$ reactivity in $50.0 \%$. Immunoreactivity to CaSR was not detectable in radiobinding assays in this same study.

Similar to non-APECED patients, thyroid peroxidase and/or thyroglobulin Abs are detectable in patients with autoimmune thyroiditis $(11,62)$.

Antibodies against pancreatic glutamic acid decarboxylase 65 (GAD65), tyrosine phosphatase-like protein IA-2 and insulin, which are prevalent in TID, may also be detected in individuals with APS-1. However, they display different sensitivity, specificity, and predictive value for diabetes. IA-2-Abs are present in a minority of APS-1 patients with TID, but they exhibit high specificity (96\%-100\%) (63). On the other hand, GAD65-Abs are common in APS-1, but they are not independently associated with TID (16). The Abs directed to GAD in APS- 1 react with different epitopes compared with the usual Abs found in TID (64). Based on such information, IA-2-Abs seem to be the best predictor for TID in APS-1 with little additive information provided by GAD65-Abs.

Endocrine cells are present throughout the gastrointestinal tract and evidence sustains the idea that gastrointestinal dysfunction in APS- 1 is actually an endocrine manifestation. Identified self-Ags include tryptophan hydroxylase (TPH) $(16,65)$, an enzyme involved in the synthesis of serotonin, and histidine decarboxylase (HDC) (66), the histamine-synthesizing enzyme. The presence of these self-Abs was correlated with loss of enterochromaffin and enterochromaffin-like cells in biopsy specimens. Self-Abs against GAD65 were also found to be associated with intestinal failure (16), what is actually reasonable, once GAD65 is highly expressed in the nervous system, including the neural plexi of the gut. The association between gastrointestinal dysfunction and loss of colecystokinin-producing cells was demonstrated in a report that described a patient with intermittent severe diarrhea and malabsorption (67). During the malabsorption period, postprandial serum cholecystokinin concentrations were not detectable, and cholecystokinin cells could not be identified in duodenal 
biopsy. However, during episodes of clinical remission, cholecystokinin concentrations increased normally in response to food, and cholecystokinin cells were identified. Nevertheless, the authors could not identify Abs against enteroendocrine- or cholecystokinin-producing cells. Cholecystokinin deficiency as the main cause of the patient's malabsorption is still questionable.

Cytochromes P450 2A6 (CYP2A6), and mainly P450 1A2 (CYPlA2), are enzymes that have been reported as self-Ags in patients with autoimmune hepatitis $(62,68)$; aromatic 1 -amino acid decarboxylase (AADC) has also been implicated (68). Other possible self-Ags are the enzymes TPH (16) and HDC (66), which are also associated with gastrointestinal dysfunction, as stated above.

The markers of autoimmune gastritis in APS- 1 are the same found in general population, which are Abs to parietal cells $\left(\mathrm{H}^{+} \mathrm{K}^{+}\right.$ATPase $)$and to intrinsic factor (11).

Researchers have recently identified a putative potassium channel regulator (KCNRG) as a pulmonary self-Ag (27). Self-Abs against KCNRG were detected in 7 of the 8 patients with respiratory symptoms, but in only 1 of 102 APS-1 patients without respiratory manifestations. Therefore, these findings bring out the idea that pulmonary involvement can be considered another autoimmune manifestation of the syndrome.

Self-antibodies to pituitary proteins, such as the tudor domain-containing protein 6 (TDRD6), were also identified, but they do not seem to be associated with clinical hypopituitarism or hypophysitis in APS-1 patients (69).

Some patients with APS- 1 and vitiligo exhibit complement-fixing melanocyte Abs (11), as well as Abs against the transcription factors SOX9 and SOXI0 (70). AADC self-Abs were also found to be associated with vitiligo (16), but the relevance of these Abs in the syndrome is still uncertain.

Self-Abs to tyrosine hydroxylase (TH) (71), an enzyme involved in the synthesis of dopamine, and to keratinocytes of the hair follicles (72) were found in patients with alopecia.

As previously stated, APS-1 patients may display neutralizing self-Abs against IL-17A, IL-17F, and IL22 cytokines $(50,51)$. This is thought to be the cause of the susceptibility to CMC, since it was noted that IL-17F and IL-22 responses to Candida albicans Ags were severely reduced. In those two reports, the levels of such Abs were low or undetectable in healthy subjects and unaffected relatives.
Besides the tissue-related self-Abs, it has been found that APS-1 patients virtually always develop high-titer neutralizing type I IFN-alpha and IFN-omega self-Abs $(6,13)$. Type I IFNs are known to be antiviral and antiproliferative cytokines, and they also stimulate cytotoxic activity in a variety of cells of the immune system. Titers of self-Abs to anti-INF are almost always high initially and persist for decades, regardless of the exact AIRE mutation, gender, ethnic background, and disease components. In contrast, these Abs could not be detected in healthy subjects, unaffected relatives, controls with other infectious, autoimmune or neoplastic diseases, with the exception of thymoma and myasthenia gravis. Their sensitivity, specificity, and predictive values exceed 98\%. Therefore, it has been suggested that IFNalpha and IFN-omega self-Abs should be included as additional diagnostic criteria for APS-1, provided thymoma and myasthenia gravis are excluded.

\section{TREATMENT}

The mainstream treatment of this syndrome is to supply the various deficiencies, which, at first, may seem a simple procedure. However, tricky situations are likely to arise, which require utmost attention and are detailed below.

Patients suffering from HP must receive calcium supplement and vitamin $\mathrm{D}$ derivatives in a dose sufficient to maintain plasma calcium in the lower half or slightly below the normal range, and urinary calcium in the normal range.

As a life-threatening condition, AI should be diagnosed before it turns symptomatic. Glucocorticoid replacement must be initiated immediately and doses have to be increased during periods of acute stress, like infections and surgery. It is important to be aware of the likelihood of hypocalcemia whenever the glucocorticoid replacement is increased, and to adjust hypoparathyroidism treatment accordingly. Mineralocorticoid deficiency is managed with fludrocortisone acetate.

L-thyroxin supplementation is administered in case of hypothyroidism, always after ruling out or treating AI. Thyroid hormone increases hepatic clearance of cortisol, and may precipitate adrenal crisis in undiagnosed AI. In addition, patients with non-treated AI may display a reversible increase in thyrotropin levels, since glucocorticoids inhibit thyrotropin secretion.

Estrogen or androgen replacement should start at pubertal age in hypogonadal children. Doses should 
be gradually increased, and treatment should be maintained during adulthood.

Vitamin B12 replacement therapy should be given whenever necessary.

It is noteworthy that intestinal dysfunction may lead to inadequate and erratic absorption of medications. Maintaining appropriate levels of hormones and other substances may be really challenging in some cases. Malabsorption may be improved with oral replacement of pancreatic enzymes and by decreasing fat intake or replacing common fat by medium-chain triglycerides (14). Again, it must be emphasized that hypocalcemia may worsen malabsorption and, therefore, must be prevented.

CMC should be controlled with anti-fungal medication. Azole agents like miconazole, ketoconazole, itraconazole, and fluconazole are often effective, although long-term use may lead to drug resistance $(73,74)$. Another important issue to be remembered is that azoles may cause defective steroidogenesis, worsening or causing AI. Amphotericin B and nystatin, polyene class antifungals, should be preferred. Oral and esophageal candidiasis must be strictly controlled to prevent development of squamous cell carcinoma (75).

Keratitis may be ameliorated with local glucocorticoids, and topical vitamin A may prevent corneal ulcerations (22). Corneal transplantation and treatment with limbal stem cells have been performed in few cases $(28,76,77)$.

Patients with asplenia are recommended to be vaccinated against pneumococcus, but some authors suggest that every APS-1 patient should be vaccinated, since asplenia is commonly asymptomatic (14).

The role of immunosuppressive therapy has been reported in several publications, with different drugs. Immunosuppressive therapy seems to control malabsorption and pancreatic insufficiency, hepatitis, keratitis, alopecia, and other symptoms, but with variable results $(12,22)$. A young patient with tubulointerstitial nephritis and renal insufficiency received tacrolimus, mycophenolate mofetil, and prednisolone after kidney transplant. These medications led to stable renal function and improved hypoparathyroidism, candidiasis, thyroiditis, thrombocytopenia, alopecia, and Sjögren syndrome, with normalization of self-Ab levels. However, exocrine pancreatic insufficiency persisted, and anti-intrinsic factor Abs remained positive (25). Another patient was given cyclosporine-A and presented improvement of pancreatic insufficiency, alopecia, and keratitis, but her self-Ab levels did not decrease (22). It is important to remember that immunosuppressive therapy predispose the patient to the spreading of superficial candidiasis to a generalized and severe form $(4,11,12)$.

\section{DISCUSSION}

In this paper, we report an emblematic case of APS-1 that displayed several features described in the literature. The patient presented the first symptoms during childhood, as the majority of the affected individuals. The three major components developed in the characteristic chronological order: candidiasis was the first to appear when she was 5 years old, and symptoms of hypocalcemia appeared at the age of 17 , although we believe hypoparathyroidism was already present months or even years before that. To date, the patient has not developed clinical adrenal insufficiency, even though tests revealed subclinical disease. Candidiasis affects her oral mucosa, skin and nails; as expected, the generalized disease has not developed, since she has never been under immunosuppressive treatment. Like 12\%$60 \%$ of the APS-1 patients, this patient suffers from hypergonadotropic hypogonadism, and similar to the majority of the patients, she has enamel hypoplasia. Besides this teeth disorder, ectodermal dysplasia also appears in her nails and corneas. Although there are only few reports about the treatment of keratopathy with stem cell transplantation, the patient has undergone the procedure with successful results. Some infrequent components of the syndrome, reported only in sporadic cases, are also present: tubulointerstitial nephritis and bronchiectasis. The patient also displays impaired fasting glucose, however the only detectable antibody is the one against GAD65. Based on the findings that GAD65-Abs do not seem to be a good predictor of TID in APS-1, and that they are found to be associated with intestinal failure, we believe that, in this case, these Abs represent autoimmune gastrointestinal disease, since her malabsorption had no other clear cause. As stated before, treatment may be extremely challenging. In this particular case, the most difficult issue is how to maintain appropriate levels of hormones, once because of malabsorption medications must be administered in uncomfortable high doses.

\section{CONCLUSION}

APS- 1 is a complex syndrome characterized by multiple-organ damage. Although rare, it must be diagnosed 
in early stages, given its high morbidity and mortality. Treatment and follow-up are demanding and require a multidisciplinary approach. The main objective is to preserve the patient's quality of life and psychosocial support is essential for most patients. Physicians should be able to promptly recognize and treat new disease components, which may develop throughout the patient's lifetime.

Acknowledgement: we are grateful to the physicians of the Bone and Mineral Unit-Unifesp. The author was supported by a research grant of Coordenação de Aperfeiçoamento de Pessoal de Nível Superior (Capes).

Disclosure: no potential conflict of interest relevant to this article was reported.

\section{REFERENCES}

1. Dittmar M, Kahaly GJ. Polyglandular autoimmune syndromes: immunogenetics and long-term follow-up. J Clin Endocrinol Metab. 2003;88(7):2983-92.

2. Neufeld MB, Blizzard RM. Polyglandular autoimmune diseases. In: Pinchera A, Doniach D, Fenzi GF, Baschieri L, eds. Symposium on autoimmune aspects of endocrine disorders. New York: Academic Press; 1980. p. 357-65.

3. Neufeld M, Maclaren NK, Blizzard RM. Two types of autoimmune Addison's disease associated with different polyglandular autoimmune (PGA) syndromes. Medicine. 1981;60(5):355-62.

4. Ahonen P, Myllarniemi S, Sipila I, Perheentupa J. Clinical variation of autoimmune polyendocrinopathy-candidiasis-ectodermal dystrophy (APECED) in a series of 68 patients. New Engl J Med. 1990;322(26):1829-36.

5. Neufeld M, Maclaren N, Blizzard R. Autoimmune polyglandular syndromes. Pediatr Ann. 1980;9(4):154-62.

6. Meloni A, Furcas M, Cetani F, Marcocci C, Falorni A, Perniola R, et al. Autoantibodies against type I interferons as an additional diagnostic criterion for autoimmune polyendocrine syndrome type I. J Clin Endocrinol Metab. 2008;93(11):4389-97. Epub 2008 Aug 26.

7. Thorpe ES, Handley HE. Chronic tetany and chronic mycelial stomatitis in a child aged 4 and half years. Am J Dis Child. 1929;38(2):328-38.

8. Leonard MF. Chronic idiopathic hypoparathyroidism with superimposed Addison's disease in a child. J Clin Endocrinol Metab. 1946;6:493-506.

9. Zlotogora J, Shapiro MS. Polyglandular autoimmune syndrome type I among Iranian Jews. J Med Genet. 1992;29(11):824-6.

10. Rosatelli MC, Meloni A, Devoto M, Cao A, Scott HS, Peterson P, et al. A common mutation in Sardinian autoimmune polyendocrinopathy-candidiasis-ectodermal dystrophy patients. Human genetics. 1998;103(4):428-34.

11. Betterle C, Greggio NA, Volpato M. Clinical review 93: autoimmune polyglandular syndrome type 1. J Clin Endocrinol Metab. 1998;83(4):1049-55.

12. Perheentupa J. Autoimmune polyendocrinopathy-candidiasis-ectodermal dystrophy. J Clin Endocrinol Metab. 2006;91(8):2843-50.

13. Meager A, Visvalingam K, Peterson P, Moll K, Murumagi A, Krohn $K$, et al. Anti-interferon autoantibodies in autoimmune polyendocrinopathy syndrome type 1. PLoS Med. 2006;3(7):e289.
14. Perheentupa J. APS-I/APECED: the clinical disease and therapy. Endocrinol Metab Clin North Am. 2002;31(2):295-320, vi.

15. Vogel A, Strassburg CP, Obermayer-Straub P, Brabant G, Manns MP.The genetic background of autoimmune polyendocrinopathy-candidiasis-ectodermal dystrophy and its autoimmune disease components. J Mol Med (Berl). 2002;80(4):201-11.

16. Soderbergh A, Myhre AG, Ekwall O, Gebre-Medhin G, Hedstrand $H$, Landgren $E$, et al. Prevalence and clinical associations of 10 defined autoantibodies in autoimmune polyendocrine syndrome type I. J Clin Endocrinol Metab. 2004;89(2):557-62.

17. Gylling M, Kaariainen E, Vaisanen R, Kerosuo L, Solin ML, Halme $\mathrm{L}$, et al. The hypoparathyroidism of autoimmune polyendocrinopathy-candidiasis-ectodermal dystrophy protective effect of male sex. J Clin Endocrinol Metab. 2003;88(10):4602-8.

18. Cetani F, Barbesino G, Borsari S, Pardi E, Cianferotti L, Pinchera $A$, et al. A novel mutation of the autoimmune regulator gene in an Italian kindred with autoimmune polyendocrinopathy-candidiasis-ectodermal dystrophy, acting in a dominant fashion and strongly cosegregating with hypothyroid autoimmune thyroiditis. J Clin Endocrinol Metab. 2001;86(10):4747-52.

19. Halonen M, Eskelin P, Myhre AG, Perheentupa J, Husebye ES, Kampe $\mathrm{O}$, et al. AIRE mutations and human leukocyte antigen genotypes as determinants of the autoimmune polyendocrinopathy-candidiasis-ectodermal dystrophy phenotype. J Clin Endocrinol Metab. 2002;87(6):2568-74.

20. Queiroz MS. [Type 1 diabetes and autoimmune polyendocrine syndromes]. Arq Bras Endocrinol Metabol. 2008;52(2):198-204.

21. Dib SA. [Heterogeneity of type 1 diabetes mellitus]. Arq Bras Endocrinol Metabol. 2008;52(2):205-18.

22. Ward L, Paquette J, Seidman E, Huot C, Alvarez F, Crock P, et al. Severe autoimmune polyendocrinopathy-candidiasis-ectodermal dystrophy in an adolescent girl with a novel AIRE mutation: response to immunosuppressive therapy. J Clin Endocrinol Metab. 1999;84(3):844-52.

23. Heubi JE, Partin JC, Schubert WK. Hypocalcemia and steatorrhea--clues to etiology. Dig Dis Sci. 1983;28(2):124-8.

24. Friedman TC, Thomas PM, Fleisher TA, Feuillan P, Parker RI, Cassorla $F$, et al. Frequent occurrence of asplenism and cholelithiasis in patients with autoimmune polyglandular disease type I. Am J Med. 1991;91(6):625-30.

25. Ulinski T, Perrin L, Morris M, Houang M, Cabrol S, Grapin C, et al. Autoimmune polyendocrinopathy-candidiasis-ectodermal dystrophy syndrome with renal failure: impact of posttransplant immunosuppression on disease activity. J Clin Endocrinol Metab. 2006;91(1):192-5.

26. García-Hernández FJ, Ocaña-Medina C, González-León R, Garrido-Rasco R, Sánchez-Román J. Autoimmune polyglandular syndrome and pulmonary arterial hypertension. Eur Respir J. 2006;27(3):657-8.

27. Alimohammadi M, Dubois N, Skoldberg F, Hallgren A, Tardivel I, Hedstrand $\mathrm{H}$, et al. Pulmonary autoimmunity as a feature of autoimmune polyendocrine syndrome type 1 and identification of KCNRG as a bronchial autoantigen. Proc Natl Acad Sci U S A. 2009;106(11):4396-401. Epub 2009 Feb 26.

28. Shah M, Holland E, Chan CC. Resolution of autoimmune polyglandular syndrome-associated keratopathy with keratolimbal stem cell transplantation: case report and historical literature review. Cornea. 2007;26(5):632-5.

29. Merenmies L, Tarkkanen A. Chronic bilateral keratitis in autoimmune polyendocrinopathy-candidiadis-ectodermal dystrophy (APECED). A long-term follow-up and visual prognosis. Acta Ophthalmol Scand. 2000;78(5):532-5.

30. Harris M, Kecha O, Deal C, Howlett CR, Deiss D, Tobias V, et al. Reversible metaphyseal dysplasia, a novel bone phenotype, in two unrelated children with autoimmunepolyendocrinopathy- 
-candidiasis-ectodermal dystrophy: clinical and molecular studies. J Clin Endocrinol Metab. 2003;88(10):4576-85.

31. Sato K, Nakajima K, Imamura H, Deguchi T, Horinouchi S, Yamazaki $\mathrm{K}$, et al. A novel missense mutation of AIRE gene in a patient with autoimmune polyendocrinopathy, candidiasis and ectodermal dystrophy (APECED), accompanied with progressive muscular atrophy: case report and review of the literature in Japan. Endocr J. 2002;49(6):625-33.

32. Nagamine K, Peterson P, Scott HS, Kudoh J, Minoshima S, Heino $M$, et al. Positional cloning of the APECED gene. Nat Genet. 1997;17(4):393-8.

33. Finnish-German APECED Consortium. An autoimmune disease, APECED, caused by mutations in a novel gene featuring two PHD-type zinc-finger domains. Nat Genet. 1997;17(4):399-403.

34. Gibson TJ, Ramu C, Gemund C, Aasland R. The APECED polyglandular autoimmune syndrome protein, AIRE-1, contains the SAND domain and is probably a transcription factor. Trends Biochem Sci. 1998;23(7):242-4.

35. Pitkanen J, Doucas V, SternsdorfT, Nakajima T, Aratani S, Jensen $\mathrm{K}$, et al. The autoimmune regulator protein has transcriptional transactivating properties and interacts with the common coactivator CREB-binding protein. J Biol Chem. 2000;275(22):16802-9.

36. Ferguson BJ, Alexander C, Rossi SW, Liiv I, Rebane A, Worth CL, et al. AIRE's CARD revealed, a new structure for central tolerance provokes transcriptional plasticity. J Biol Chem. 2008;283(3):172331. Epub 2007 Nov 1.

37. Pitkanen J, Vahamurto P, Krohn K, Peterson P. Subcellular localization of the autoimmune regulator protein. characterization of nuclear targeting and transcriptional activation domain. $\mathrm{J}$ Biol Chem. 2001;276(22):19597-602. Epub 2001 Mar 26.

38. Plevin MJ, Mills MM, Ikura M. The LxxLL motif: a multifunctional binding sequence in transcriptional regulation. Trends Biochem Sci. 2005;30(2):66-9.

39. Bienz M. The PHD finger, a nuclear protein-interaction domain. Trends Biochem Sci. 2006;31(1):35-40. Epub 2005 Nov 16.

40. Uchida D, Hatakeyama S, Matsushima A, Han H, Ishido S, Hotta $\mathrm{H}$, et al. AlRE functions as an E3 ubiquitin ligase. J Exp Med. 2004;199(2):167-72.

41. Bottomley MJ, Stier G, Pennacchini D, Legube G, Simon B, Akhtar $A$, et al. NMR structure of the first PHD finger of autoimmune regulator protein (AIRE1). Insights into autoimmune polyendocrinopathy-candidiasis-ectodermal dystrophy (APECED) disease. $J$ Biol Chem. 2005;280(12):11505-12.

42. Anderson MS, Venanzi ES, Klein L, Chen Z, Berzins SP, Turley SJ, et al. Projection of an immunological self shadow within the thymus by the aire protein. Science. 2002;298(5597):1395-401. Epub 2002 Oct 10.

43. Kogawa K, Nagafuchi S, Katsuta H, Kudoh J, Tamiya S, SakaiY, et al. Expression of AIRE gene in peripheral monocyte/dendritic cell lineage. Immunol Lett. 2002;80(3):195-8.

44. Gray D, Abramson J, Benoist C, Mathis D. Proliferative arrest and rapid turnover of thymic epithelial cells expressing Aire. J Exp Med. 2007;204(11):2521-8. Epub 2007 Oct 1.

45. Kekalainen E, Tuovinen H, Joensuu J, Gylling M, Franssila R, Pontynen $\mathrm{N}$, et al. A defect of regulatory $\mathrm{T}$ cells in patients with autoimmune polyendocrinopathy-candidiasis-ectodermal dystrophy. J Immunol. 2007;178(2):1208-15.

46. Kuroda N, Mitani T, Takeda N, Ishimaru N, Arakaki R, Hayashi Y, et al. Development of autoimmunity against transcriptionally unrepressed target antigen in the thymus of Aire-deficient mice. J Immunol. 2005;174(4):1862-70.

47. Pontynen N, Strengell M, Sillanpaa N, Saharinen J, Ulmanen I, Julkunen I, et al. Critical immunological pathways are down- regulated in APECED patient dendritic cells. $\mathrm{J}$ Mol Med (Berl). 2008;86(10):1139-52.

48. Perniola R, Congedo M, Rizzo A, Sticchi Damiani A, Faneschi ML, Pizzolante $\mathrm{M}$, et al. Innate and adaptive immunity in patients with autoimmune polyendocrinopathy-candidiasis-ectodermal dystrophy. Mycoses. 2008;51(3):228-35.

49. Conti HR, Shen F, Nayyar N, Stocum E, Sun JN, Lindemann MJ, et al. Th17 cells and IL-17 receptor signaling are essential for mucosal host defense against oral candidiasis. J Exp Med. 2009;206(2):299-311.

50. Kisand K, Boe Wolff AS, Podkrajsek KT, Tserel L, Link M, Kisand $\mathrm{KV}$, et al. Chronic mucocutaneous candidiasis in APECED or thymoma patients correlates with autoimmunity to Th17-associated cytokines. J Exp Med. 2010;207(2):299-308.

51. Puel A, Doffinger R, Natividad A, Chrabieh M, Barcenas-Morales G, Picard C, et al. Autoantibodies against IL-17A, IL-17F, and IL-22 in patients with chronic mucocutaneous candidiasis and autoimmune polyendocrine syndrome type I. J Exp Med. 2010;207(2):291-7.

52. Su MA, Giang K, Zumer K, Jiang H, Oven I, Rinn JL, et al. Mechanisms of an autoimmunity syndrome in mice caused by a dominant mutation in Aire. J Clin Invest. 2008;118(5):1712-26.

53. Bjorses $\mathrm{P}$, Halonen $\mathrm{M}$, Palvimo JJ, Kolmer M, Aaltonen J, Ellonen $P$, et al. Mutations in the AIRE gene: effects on subcellular location and transactivation function of the autoimmune polyendocrinopathy-candidiasis-ectodermal dystrophy protein. Am J Hum Genet. 2000;66(2):378-92.

54. Wolff AS, Erichsen MM, Meager A, Magitta NF, Myhre AG, Bollerslev $\mathrm{J}$, et al. Autoimmune polyendocrine syndrome type 1 in Norway: phenotypic variation, autoantibodies, and novel mutations in the autoimmune regulator gene. J Clin Endocrinol Metabol. 2007;92(2):595-603.

55. Pearce SH, Cheetham T, Imrie H, Vaidya B, Barnes ND, Bilous RW, et al. A common and recurrent 13-bp deletion in the autoimmune regulator gene in British kindreds with autoimmune polyendocrinopathy type 1. Am J Hum Genet. 1998;63(6):1675-84.

56. Heino M, Scott HS, Chen Q, Peterson P, Maebpaa U, Papasavvas MP, et al. Mutation analyses of North American APS-1 patients. Hum Mutat. 1999;13(1):69-74.

57. Meriluoto $T$, Halonen $M$, Pelto-Huikko $M$, Kangas $H$, Korhonen $\mathrm{J}$, Kolmer $\mathrm{M}$, et al. The autoimmune regulator: a key toward understanding the molecular pathogenesis of autoimmune polyendocrinopathy-candidiasis-ectodermal dystrophy. Keio J Med. 2001;50(4):225-39.

58. Chen S, Sawicka J, Betterle C, Powell M, Prentice L, Volpato M, et al. Autoantibodies to steroidogenic enzymes in autoimmune polyglandular syndrome, Addison's disease, and premature ovarian failure. J Clin Endocrinol Metab. 1996;81(5):1871-6.

59. Reimand K, Perheentupa J, Link M, Krohn K, Peterson P, Uibo R. Testis-expressed protein TSGA10 an auto-antigen in autoimmune polyendocrine syndrome type I. Int Immunol. 2008;20(1):39-44. Epub 2007 Nov 13.

60. Alimohammadi M, Bjorklund P, Hallgren A, Pontynen N, Szinnai G, Shikama N, et al. Autoimmune polyendocrine syndrome type 1 and NALP5, a parathyroid autoantigen. N Engl J Med. 2008 Mar 6;358(10):1018-28.

61. Gavalas NG, Kemp EH, Krohn KJ, Brown EM, Watson PF, Weetman AP. The calcium-sensing receptor is a target of autoantibodies in patients with autoimmune polyendocrine syndrome type 1. J Clin Endocrinol Metab. 2007;92(6):2107-14. Epub 2007 Mar 20.

62. Perniola R, Falorni A, Clemente MG, Forini F, Accogli E, Lobreglio G. Organ-specific and non-organ-specific autoantibodies in children and young adults with autoimmune polyendocrinopathy-candidiasis-ectodermal dystrophy (APECED). Eur J Endocrinol. 2000;143(4):497-503. 
63. Gylling M, Tuomi T, Bjorses $\mathrm{P}$, Kontiainen S, Partanen J, Christie $\mathrm{MR}$, et al. ss-cell autoantibodies, human leukocyte antigen II alleles, and type 1 diabetes in autoimmune polyendocrinopathy-candidiasis-ectodermal dystrophy. J Clin Endocrinol Metab. 2000 Dec;85(12):4434-40.

64. TuomiT, Bjorses P, Falorni A, Partanen J, Perheentupa J, Lernmark $A$, et al. Antibodies to glutamic acid decarboxylase and insulin-dependent diabetes in patients with autoimmune polyendocrine syndrome type I. J Clin Endocrinol Metab. 1996;81(4):1488-94.

65. Ekwall O, Hedstrand H, Grimelius L, Haavik J, Perheentupa J, Gustafsson J, et al. Identification of tryptophan hydroxylase as an intestinal autoantigen. Lancet. 1998;352(9124):279-83.

66. Skoldberg F, Portela-Gomes GM, Grimelius L, Nilsson G, Perheentupa J, Betterle C, et al. Histidine decarboxylase, a pyridoxal phosphate-dependent enzyme, is an autoantigen of gastric enterochromaffin-like cells. J Clin Endocrinol Metab. 2003;88(4): 1445-52.

67. Hogenauer C, Meyer RL, Netto GJ, Bell D, Little KH, Ferries L, et al. Malabsorption due to cholecystokinin deficiency in a patient with autoimmune polyglandular syndrome type I. New Engl J Med. $2001 ; 344(4): 270-4$

68. Gebre-Medhin G, Husebye ES, Gustafsson J, Winqvist O, Goksoyr A, Rorsman F, et al. Cytochrome P450IA2 and aromatic L-amino acid decarboxylase are hepatic autoantigens in autoimmune polyendocrine syndrome type I. FEBS Letters. 1997;412(3):439-45.

69. Bensing S, Fetissov SO, Mulder J, Perheentupa J, Gustafsson $\mathrm{J}$, Husebye ES, et al. Pituitary autoantibodies in autoimmune polyendocrine syndrome type 1. Proc Natl Acad Sci U S A. 2007;104(3):949-54. Epub 2007 Jan 10.

70. Hedstrand H, Ekwall O, Olsson MJ, Landgren E, Kemp EH, Weetman AP, et al. The transcription factors SOX9 and SOX10 are vitili- go autoantigens in autoimmune polyendocrine syndrome type I. J Biol Chem. 2001;276(38):35390-5.

71. Hedstrand H, Ekwall O, Haavik J, Landgren E, Betterle C, Perheentupa $\mathrm{J}$, et al. Identification of tyrosine hydroxylase as an autoantigen in autoimmune polyendocrine syndrome type I. Biochem Biophys Res Commun. 2000;267(1):456-61.

72. Hedstrand H, Perheentupa J, Ekwall O, Gustafsson J, Michaelsson $G$, Husebye $E$, et al. Antibodies against hair follicles are associated with alopecia totalis in autoimmune polyendocrine syndrome type I. J Invest Dermatol. 1999;113(6):1054-8.

73. Rautemaa R, Richardson M, Pfaller M, Koukila-Kahkola P, Perheentupa J, Saxen H. Decreased susceptibility of Candida albicans to azole antifungals: a complication of long-term treatment in autoimmune polyendocrinopathy-candidiasis-ectodermal dystrophy (APECED) patients. J Antimicrob Chemother. 2007;60(4):889-92. Epub 2007 Aug 17.

74. Siikala E, Rautemaa R, Richardson M, Saxen H, Bowyer P, Sanglard D. Persistent Candida albicans colonization and molecular mechanisms of azole resistance in autoimmune polyendocrinopathy-candidiasis-ectodermal dystrophy (APECED) patients. J Antimicrob Chemother. 2010;65(12):2505-13. Epub 2010 Sep 28.

75. Rautemaa R, Hietanen J, Niissalo S, Pirinen S, Perheentupa J. Oral and oesophageal squamous cell carcinoma--a complication or component of autoimmune polyendocrinopathy-candidiasis-ectodermal dystrophy (APECED, APS-I). Oral Oncol. 2007;43(6):607-13. Epub 2006 Sep 25.

76. Tarkkanen A, Merenmies L. Corneal pathology and outcome of keratoplasty in autoimmune polyendocrinopathy-candidiasis-ectodermal dystrophy (APECED). Acta Ophthalmol Scand. 2001;79(2):204-7.

77. Meller D, Pires RT, Tseng SC. Ex vivo preservation and expansion of human limbal epithelial stem cells on amniotic membrane cultures. Br J Ophthalmol. 2002;86(4):463-71. 